\title{
Solar-Powered Aircraft: Energy-Optimal Path Planning and Perpetual Endurance
}

\author{
Andrew T. Klesh $*$ and Pierre T. Kabamba ${ }^{\ddagger}$ \\ University of Michigan, Ann Arbor, Michigan, 48109
}

DOI: $\underline{10.2514 / 1.40139}$

\begin{abstract}
This paper considers energy-optimal path planning and perpetual endurance for unmanned aerial vehicles equipped with solar cells on the wings, which collect energy used to drive a propeller. Perpetual endurance is the ability to collect more energy than is lost during a day. This paper considers two unmanned aerial vehicle missions: 1) to travel between given positions within an allowed duration while maximizing the final value of energy and 2) to loiter perpetually from a given position, which requires perpetual endurance. For the first mission, the subsequent problem of energy-optimal path planning features the coupling of the aircraft kinematics and energetics models through the bank angle. The problem is then formulated as an optimal control problem, with the bank angle and speed as inputs. Necessary conditions for optimality are formulated and used to study the optimal paths. The power ratio, a nondimensional number, is shown to predict the qualitative features of the optimal paths. This ratio also quantifies a design requirement for the second mission. Specifically, perpetual endurance is possible if and only if the power ratio exceeds a certain threshold. Comparisons are made of this threshold between Earth and Mars. Implications of the power ratio for unmanned aerial vehicle design are also discussed. Several illustrations are given.
\end{abstract}

\section{Nomenclature}

$C_{D}$

$C_{D_{o}}$

$C_{L}$

D

$E_{R}$

$E_{T}$

$E_{\text {in }}$

$E_{\text {out }}$

e

$g$

H

$H_{V V}$

$H_{\phi V}$

$H_{\phi \phi}$

$i$

j

K

$m$

$P_{R}$

$P_{\text {in }}$

$P_{\text {out }}$

$P_{\text {sd }}$

S

$T$

$T_{M}$

$t_{f}$

$t_{o}$

V

$V_{\text {energy }}$ min

$V_{\text {power }_{\text {min }}}$

$x$

W

$=$ azimuth of the sun, deg

$=$ coefficient of drag

$=$ parasitic drag coefficient

$=$ coefficient of lift

$=$ drag of the aircraft, $\mathrm{N}$

$=$ energy ratio

$=$ total energy, $\mathrm{J}$

$=$ energy collected, $\mathrm{J}$

$=$ energy lost, $\mathrm{J}$

$=$ elevation of the sun, deg

$=$ Hamiltonian, $\mathrm{W}$

$=\partial^{2} H / \partial V^{2}$

$=\partial^{2} H / \partial \phi \partial V$

$=\partial^{2} H / \partial \phi^{2}$

$=$ dummy summation index an elliptical lift distribution

$=$ mass, $\mathrm{kg}$

$=$ power ratio

$=$ power collected, $\mathrm{W}$

= power lost, $\mathrm{W}$

$=$ surface area of the wing, $\mathrm{m}^{2}$

$=$ thrust of the aircraft, $\mathrm{N}$

$=$ mission time, $\mathrm{s}$

$=$ final time, $\mathrm{s}$

$=$ initial time, $\mathrm{s}$

$=$ speed, $\mathrm{m} / \mathrm{s}$

$=X$ position, $\mathrm{m}$

$=$ weight of the aircraft, $\mathrm{N}$
$=$ gravitational acceleration, $\mathrm{m} / \mathrm{s}^{2}$

$=$ incidence angle of sun rays, deg

$=$ amount whereby the induced drag exceeds that of

$=$ power spectral density of the sun, $\mathrm{W} / \mathrm{m}^{2}$

$=$ speed at minimum energy out, $\mathrm{m} / \mathrm{s}$

$=$ speed at minimum power out, $\mathrm{m} / \mathrm{s}$
Received 30 July 2008; accepted for publication 19 January 2009. Copyright (C) 2009 by the American Institute of Aeronautics and Astronautics, Inc. All rights reserved. Copies of this paper may be made for personal or internal use, on condition that the copier pay the $\$ 10.00$ per-copy fee to the Copyright Clearance Center, Inc., 222 Rosewood Drive, Danvers, MA 01923; include the code 0731-5090/09 \$10.00 in correspondence with the CCC.

*Graduate Student, Aerospace Engineering, 1320 Beal Avenue.

${ }^{\dagger}$ Professor, Aerospace Engineering, 1320 Beal Avenue.

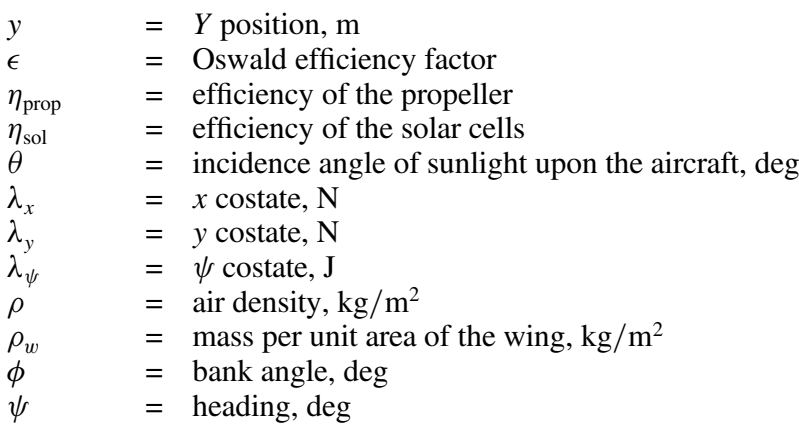

\section{Introduction}

$\mathbf{F}$ UTURE exploration of Mars, laid out by the Vision for Space Exploration [1], requires long-endurance unmanned aerial vehicles (UAVs) that use resources that are plentiful on Mars. One possible way of achieving this is to use solar-powered UAVs that fly perpetually, which motivates the problems solved in this paper. The UAVs considered in this paper are distinguished from the majority of UAVs by their power source: they are equipped with solar cells on the upper surface of the wings as well as onboard energy storage. These solar cells collect energy that is used to drive a propeller.

This paper considers the problem of energy-optimal path planning for solar-powered UAVs in level flight and quantifies the requirement for perpetual endurance in solar-powered flight. Perpetual endurance is the ability of a UAV to collect more energy from the sun than it loses in flying during a solar day. These problems feature the interaction between three subsystems: aircraft kinematics, energy collection, and energy loss. Although the current literature discusses methods to optimize UAV aerodynamic design for energy usage, there is no approach that examines the coupling of energy collection and energy loss with the aircraft kinematics, and there is no specific quantification of the requirement for perpetual endurance of solarpowered flight in terms of aircraft and environmental parameters. Consequently, the purpose of this paper is twofold: 1) to investigate the coupling between energy collection, energy loss, and kinematics and to account for it in optimal path planning and 2) to identify the requirement for perpetual endurance and show its applicability to solar-powered aircraft design.

Although the current literature on solar-powered UAVs does not consider energy-optimal path planning or perpetual flight, a substantial body of work is available on the analysis and design of 
solar-powered aircraft. A brief review of this literature is as follows. The feasibility of solar-powered flight is reviewed in [2,3], and the history of solar-powered flight is discussed in [4-6]. Methods for analysis and design of solar-powered aircraft are discussed in [4-28]. Specifically, the design of solar-powered aircraft has focused on geometric configuration 12. Design of full aircraft systems can be found, in particular, in $[5,7-9,12,14,16,17,19-22,24,29]$.

The use of optimal control to generate aircraft trajectories has been extensively covered in the literature by [30-33] as well as many others. Generally, the concern has been to fly in a fuel- and energyoptimal manner [34-36] or in a time-optimal manner [37]. Multiplecost optimization (in this case, for fuel and time) is discussed in $[38,39]$. Various methods have been employed to optimize aircraft trajectories, including necessary conditions [40] and parameter reduction [41].

Optimization for solar-powered aircraft is discussed in the literature. Most path planning has only considered minimum power consumption during level flight [] $]$. References [14,17,27] use an optimization procedure to design the aircraft based upon expected maneuvers and sunlight availability. Mission design is found in $[28,42-44]$, with particular emphasis on where and when to fly. In most references, efficiency through preliminary design is emphasized. Alternative methods to increase efficiency for solarpowered aircraft are discussed in [45-47]. Reference [47] achieves a $30 \%$ increase in efficiency by improving the cooling of solar cells. Energy-efficient flight is discussed in the literature on dynamic soaring [48-50] and manned gliders [51,52]. However, nowhere in the literature is there a study optimizing the flight path itself based upon the interaction of kinematics and solar energetics or a design requirement for perpetual endurance for solar-powered flight.

The present paper presents an integrated model of the aircraft kinematics and energetics that has the following original features. First, the energy collected and lost depend upon the bank angle of the aircraft and the position of the sun. The turn rate of the aircraft is also dependent upon the bank angle. Thus, the aircraft kinematics and energetics are coupled through the bank angle. Second, the sun is not assumed to always be present in the sky or to be stationary. Maneuvers in all light conditions are considered and several regimes of flight are discussed.

Based on the integrated model, the problem of solar-powered UAV level-flight path planning is formulated as an optimal control problem, with the bank angle and speed serving as inputs. The present paper studies this optimization problem and provides the following original contributions:

1) The necessary conditions for optimality are formulated.

2) From these necessary conditions, the properties of energyoptimal paths are derived.

3) Two regimes of optimal flight are identified. The so-called power ratio, a dimensionless parameter that can be computed before flight, is shown to correctly predict the regime of optimal flight.

4) An analytic condition for perpetual endurance accounting for location, time of year, environment, and aircraft parameters is presented. The condition requires the power ratio to exceed a threshold: the perpetuity threshold.

5) This paper proves that the requirements for perpetual solarpowered flight on Mars are always significantly more stringent than on Earth. Hence, solar-powered UAVs for Mars exploration must satisfy tighter design specifications than their Earthbound counterparts.

The remainder of the paper is as follows. In Sec. II, the model is presented. In Sec. III, the problem of maximization of the total final energy of the UA $\bar{V}$ is formulated. In Sec. IV, the necessary conditions of optimality are used to characterize the optimal paths. Section $\mathrm{V}$ introduces the power ratio, and in Sec. VI, properties of the optimal paths are presented. In Sec. VII, the perpetuity threshold is derived and compared between Earth and Mars. Section VIII provides conclusions and discusses future work. Derivation of the solar position model, proofs of the propositions, and derivation of the first- and second-order necessary conditions are given in [53].

\section{A. Aircraft Kinematic Model}

\section{Modeling}

The bank-to-turn aircraft is assumed to fly in still air at constant altitude. We use the wind axes [54] for the aircraft, and thus the assumption of constant-altitude flight induces an assumption of zero pitch angle. The kinematic model is

$$
\begin{aligned}
& \dot{x}=V \cos \psi \\
& \dot{y}=V \sin \psi \\
& \dot{\psi}=\frac{g \tan \phi}{V}
\end{aligned}
$$

where $x$ and $y$ are the Cartesian coordinates of the aircraft, $\psi$ is the heading angle, $V$ is the speed, and $\phi$ is the bank angle.

\section{B. Energy-Collection Model}

The aircraft is equipped with solar cells mounted on the top side of the wings and gains solar energy from the sun shining on the cells. Let $a$ and $e$ represent the azimuth and elevation angles of the sun, respectively. Assuming that the wing configuration has zero dihedral angle, the incidence angle of the sun rays upon the solar cells, $\theta$, satisfies

$$
\cos (\theta)=\cos (\phi) \sin (e)-\cos (e) \sin (a-\psi) \sin (\phi)
$$

which is derived in [53]. The power collected by the aircraft is

$$
\begin{gathered}
P_{\text {in }}(\theta)=\eta_{\text {sol }} P_{\text {sd }} S \cos (\theta) \quad \text { if } \cos (\theta) \geq 0 \\
P_{\text {in }}(\theta)=0 \quad \text { if } \cos (\theta)<0
\end{gathered}
$$

where $\eta_{\text {sol }}$ is the efficiency of the solar cell, $P_{\text {sd }}$ is the solar spectral density, and $S$ is the total area of the wing. If less than a full wing is covered by solar cells or cloud cover is present, $\eta_{\text {sol }}$ can be adjusted to account for these.

During a time interval $\left[t_{o}, t_{f}\right]$, the energy collected by the aircraft is

$$
E_{\text {in }}=\int_{t_{o}}^{t_{f}} P_{\text {in }}(\theta) \mathrm{d} t
$$

\section{Energy-Loss Model}

Energy lost by the craft is derived from standard lift, drag and propulsion models assuming quasi-static equilibrium flight [54]. The constant-altitude assumption requires $L \cos (\phi)=m g$, where $L$ is the lift and $m g$ is the weight of the aircraft. The equations governing the power lost driving the propeller, $P_{\text {out }}$, are

$$
P_{\text {out }}(\phi, V)=\frac{T V}{\eta_{\text {prop }}}
$$

where

$$
\begin{gathered}
T=D \\
D=\frac{1}{2} \rho V^{2} S C_{D} \\
C_{D}=C_{D_{O}}+K C_{L}^{2} \\
C_{L}=\frac{2 m g}{\rho V^{2} S \cos \phi}
\end{gathered}
$$

$T$ is the thrust of the aircraft, $\eta_{\text {prop }}$ is the efficiency of the propeller, $D$ is the drag of the aircraft, $C_{D}$ is the coefficient of drag, $\rho$ is the atmospheric density, $C_{D_{O}}$ is the parasitic drag, the aerodynamic coefficient $K=1 / \epsilon \pi \mathrm{AR}$ represents the amount whereby the induced drag exceeds that of an elliptical lift distribution, $\mathrm{AR}=b^{2} / S$ is the aspect ratio of the wing, $\epsilon$ is the Oswald efficiency factor, and $C_{L}$ is the coefficient of lift. 
During a time interval $\left[t_{o}, t_{f}\right]$, the energy lost by the aircraft is

$$
E_{\text {out }}=\int_{t_{o}}^{t_{f}} P_{\text {out }}(\phi, V) \mathrm{d} t
$$

\section{Solar Position Model}

Assume that the aircraft flies in the atmosphere of a planet that 1) is in a circular orbit around the sun with constant angular velocity $\Omega$ and 2) has a spin axis that is inclined with respect to its orbital plane with inclination $i$ and constant spin rate $\omega$. If the aircraft flies at a location specified by latitude $\lambda$ and longitude $\gamma$, the elevation and azimuth of the sun at that location satisfy

$$
\begin{aligned}
e= & \arcsin (\sin (i) \sin (\lambda) \sin (\Omega t)-\cos (\lambda)[\cos (\Omega t) \cos (\gamma+\omega t) \\
& +\cos (i) \sin (\Omega t) \sin (\gamma+\omega t)])
\end{aligned}
$$

hence, the longitude and latitude of the aircraft are fixed during the mission. The perpetual-loiter problem is then to derive conditions on the aircraft and environment parameters introduced in Sec. II that make the mission possible.

\section{Optimal Path Planning}

In this section, we derive the necessary conditions for energyoptimal flight. The optimization problem is to maximize, with respect to the time histories of the bank angle and speed, the final energy of the solar-powered aircraft; that is,

$$
\max _{\phi(\cdot), V(\cdot)} E_{\text {total }} \triangleq\left(E_{\text {in }}-E_{\text {out }}\right)
$$

The necessary conditions for optimality for the maximization problem [Eqs. (1-16)] are derived in [30]. Here, these necessary

$$
a=\arctan \left(\frac{\cos (i) \cos (\gamma+\omega t) \sin (\Omega t)-\cos (\Omega t) \sin (\gamma+\omega t)}{\cos (\Omega t) \cos (\gamma+\omega t) \sin (\lambda)+\sin (\Omega t)[\cos (\lambda) \sin (i)+\cos (i) \sin (\lambda) \sin (\gamma+\omega t)]}\right)
$$

which are derived in [53].

By definition, sunrise is a time $t_{r}$ such that $e\left(t_{r}\right)=0$ and $\dot{e}\left(t_{r}\right)>0$. Sunset is a time $t_{s}$ such that $e\left(t_{s}\right)=0$ and $\dot{e}\left(t_{s}\right)<0$. Daylight is the duration between a sunrise and the next sunset (i.e., $t_{s}-t_{r}$ ). The solar day $t_{\mathrm{sd}}$ is the interval between two consecutive sunrises. The daylight duty cycle is the ratio between daylight and solar day: that is, $\left(t_{s}-t_{r}\right) / t_{\mathrm{sd}}$.

\section{E. Model Summary and Limitations}

In summary, the integrated model is as follows. The bank angle and speed determine the heading and the position of the aircraft through Eqs. (1-3). The bank angle together with the sun's position from Eqs. (14) and (15) determine the incidence angle through Eq. (4). The incidence angle of the sun together with the bank angle and speed determine the energy collected and lost by the aircraft during flight through Eqs. (ㅁ-ㄱ), (12), and (13), respectively.

The most significant limitations of the model [Eqs. (1-13)] are the assumptions of quasi-static equilibrium flight at constant altitude. Because these assumptions can be satisfied in practice but are restrictive, the energy optimization results presented in this paper provide conservative bounds on what can be achieved when these assumptions are violated. Removing these assumptions is the subject of future work.

\section{Problem Formulations}

This paper considers the following two types of missions with associated problems.

\section{A. Energy-Optimal Flight}

Here, the mission is to fly from a given initial location and heading $\left(x_{o}, y_{o}, \phi_{o}\right)$ to a given final location and heading $\left(x_{f}, y_{f}, \phi_{f}\right)$ departing at a given initial time $t_{o}$ and taking, at most, a given mission time $T_{M}$. We assume that $T_{M}$ is very short compared with daylight; hence, the sun is fixed in the sky during the mission. The optimal path-planning problem is then to find a flight path that accomplishes the mission in an energy-optimal manner (i.e., while maximizing the final value of aircraft energy).

\section{B. Perpetual Loiter}

Here, the mission is to fly from a given initial location and time $\left(x_{o}, y_{o}, t_{o}\right)$ so that, over the time interval $\left[t_{o}, t_{o}+t_{\mathrm{sd}}\right]$, the aircraft collects more energy from the sun than it spends flying. We assume that the aircraft speed is sufficiently small so that its longitude and latitude do not change significantly over the duration of a solar day; conditions are applied to the current problem. With states $[x, y, \psi]^{T}$ and control inputs $(\phi, V)^{T}$, the Hamiltonian is

$$
\begin{gathered}
H\left(x, y, \psi, \lambda_{x}, \lambda_{y}, \lambda_{\psi}, \phi, V\right)=P_{\text {in }}(\theta)-P_{\text {out }}(\phi, V) \\
+\lambda_{x} V \cos \psi+\lambda_{y} V \sin \psi+\lambda_{\psi} \frac{g \tan \phi}{V}
\end{gathered}
$$

where $\theta$ depends on $\phi$ and $\psi$ through Eq. (4), and $\lambda_{x}, \lambda_{y}$, and $\lambda_{\psi}$ are the costates. Here, the only control constraints are that $V>0$ and $|\phi|<\pi / s$.

Remark 1: It is not necessary to impose a tight constraint on the magnitude of the bank angle. Indeed, banking requires lifting [see Eq. (12)], lifting induces drag [see Eq. (10)], drag requires thrust [see Eq. (9)], which implies power loss [see Eq. ()ㅡ. Because the path planning aims at achieving optimal final energy, the magnitude of the bank angle is naturally limited by these phenomena.

The state equations derived from Eq. (16) are

$$
\begin{aligned}
& \dot{x}=\frac{\partial H}{\partial \lambda_{x}}=V \cos (\psi) \\
& \dot{y}=\frac{\partial H}{\partial \lambda_{y}}=V \sin (\psi)
\end{aligned}
$$

$$
\dot{\psi}=\frac{\partial H}{\partial \lambda_{\psi}}=\frac{g \tan (\phi)}{V}
$$

The costate equations are

$$
\begin{aligned}
& \dot{\lambda}_{x}=\frac{-\partial H}{\partial x}=0 \\
& \dot{\lambda}_{y}=\frac{-\partial H}{\partial y}=0
\end{aligned}
$$

$$
\begin{gathered}
\dot{\lambda}_{\psi}=\frac{-\partial H}{\partial \psi}=-\lambda_{y} V \cos \psi+\lambda_{x} V \sin \psi \\
-\eta_{\mathrm{sol}} P_{\mathrm{sd}} S \cos e \cos (a-\psi) \sin \phi
\end{gathered}
$$


The first-order optimality conditions are

$$
\begin{aligned}
\frac{\partial H}{\partial \phi} & =-\eta_{\mathrm{sol}} P_{\mathrm{sd}} S(\cos (e) \cos (\phi) \sin (a-\psi)+\sin (e) \sin (\phi)) \\
& -\frac{4 K(m g)^{2} \sin (\phi)}{\eta_{\text {prop }} \rho S V \cos ^{3}(\phi)}+\frac{g \lambda_{\psi}}{V \cos ^{2}(\phi)}=0 \\
\frac{\partial H}{\partial V} & =\lambda_{x} \cos (\psi)+\frac{8 K(m g)^{2} \sec (\phi)^{2}}{\eta_{\text {prop }} \rho S V^{2}} \\
& -\frac{3 \rho S V^{2}\left(C_{D_{o}}+\left[4 K(m g)^{2} \sec (\phi)^{2} / \rho^{2} S^{2} V^{4}\right]\right)}{2 \eta_{\text {prop }}} \\
& +\lambda_{y} \sin (\psi)-\frac{g \lambda_{\psi} \tan (\phi)}{V^{2}}=0
\end{aligned}
$$

The second-order Legrendre-Clebsch condition is that the Hessian of the Hamiltonian be negative semidefinite: that is,

$$
\frac{\partial^{2} H}{\partial(\phi, V)^{2}} \leq 0
$$

where if

$$
\begin{gathered}
\frac{\partial^{2} H}{\partial(\phi, V)^{2}}=\left[\begin{array}{ll}
H_{\phi \phi} & H_{\phi V} \\
H_{\phi V} & H_{V V}
\end{array}\right] \\
H_{\phi \phi}=\frac{\eta_{\text {prop }} \eta_{\text {sol }} \rho P_{\text {sd }} S^{2} V(-(\cos (\phi) \sin (e))+\cos (e) \sin (a-\psi) \sin (\phi))}{\eta_{\text {prop }} \rho S V} \\
+\frac{-4 K(m g)^{2} \sec (\phi)^{4}+2 \sec (\phi)^{3}\left(g \lambda_{\psi} \eta_{\text {prop }} \rho S \cos (\phi)-4 K(m g)^{2} \operatorname{sip}\right.}{\eta_{\text {prop }} \rho S V} \\
H_{\phi V}=-\frac{g \lambda_{\psi} \sec (\phi)^{2}}{V^{2}}+\frac{4 K(m g)^{2} \sec (\phi)^{2} \tan (\phi)}{\eta_{\text {prop }} \rho S V^{2}} \\
+\frac{2 g \lambda_{\psi} \tan (\phi)}{V^{3}} \\
H_{V V} \rho S V^{3}
\end{gathered}
$$$$
+\frac{-4 K(m g)^{2} \sec (\phi)^{4}+2 \sec (\phi)^{3}\left(g \lambda_{\psi} \eta_{\text {prop }} \rho S \cos (\phi)-4 K(m g)^{2} \sin (\phi)\right) \tan (\phi)}{\eta_{\text {prop }} \rho S V}
$$

We will refer to the flight paths that satisfy these first- and secondorder necessary conditions as extremal paths.

The boundary conditions for point-to-point flight are

$$
\begin{aligned}
& x\left(t_{o}\right)=x_{o} \\
& y\left(t_{o}\right)=y_{o} \\
& \psi\left(t_{o}\right)=\psi_{o} \\
& x\left(t_{f}\right)=x_{f} \\
& y\left(t_{f}\right)=y_{f} \\
& \psi\left(t_{f}\right)=\phi_{f}
\end{aligned}
$$

Because the final time is free, then we must also satisfy

$$
\left.H\left(x, y, \psi, \lambda_{x}, \lambda_{y}, \lambda_{\psi}, \phi, V\right)\right|_{t_{f}}=0, \quad t_{f}<t_{o}+T_{M}
$$

However, if $t_{f}=t_{o}+T_{M}$, Eq. (36) does not necessarily hold. respectively.
Equations (18-37) provide necessary conditions for optimality in the form of a two-point boundary-value problem.

\section{Power Ratio}

If $E_{\text {total }}<0$ in Eq. (16), we have that $P_{\text {in }}<P_{\text {out }}$, on average. Conversely, if $E_{\text {total }}>0$, we have $P_{\text {in }}>P_{\text {out }}$, on average. These conditions may be expressed as $P_{\text {in }} / P_{\text {out }}<1$ or $P_{\text {in }} / P_{\text {out }}>1$, respectively. When considering a straight unbanked flight path between an initial location and a final location, this ratio is

$$
P_{R}=\frac{2 \eta_{\text {prop }} \eta_{\text {sol }} \rho P_{\text {sd }} S^{2} V_{\text {power }_{\text {min }}} \sin (e)}{C_{D_{O}} \rho^{2} S^{2} V_{\text {power }_{\text {min }}}^{4}+4 K(m g)^{2}}
$$

where

$$
V_{\text {power }_{\min }}=\sqrt[4]{\frac{4 K W^{2}}{3 C_{D_{o}} \rho^{2} S^{2} \cos ^{2}(\phi)}}
$$

Note the distinction between $V_{\text {power }_{\text {min }}}$ and $V_{\text {energy }_{\min }}$, where

$$
V_{\text {energy }_{\min }}=\sqrt[4]{\frac{4 K W^{2}}{C_{D_{o}} \rho^{2} S^{2} \cos ^{2}(\phi)}}=1.31 V_{\text {power }_{\text {min }}}
$$

We refer to this nondimensional parameter as $P_{R}$, the power ratio.

On an energy-optimal flight, we can evaluate the energy collected and energy lost and compute another nondimensional quantity, the energy ratio $E_{R}$, as

$$
E_{R}=\frac{\int_{t_{o}}^{t_{f}} P_{\text {in }}(\theta) \mathrm{d} t}{\int_{t_{o}}^{t_{f}} P_{\text {out }}(\phi, V) \mathrm{d} t}
$$

where $E_{R}$ defines two flight regimes, which we call the drag regime and the solar regime, corresponding to $E_{R}<1$ and $E_{R}>1$,

Although $P_{R}$ does not depend on $(\phi, \psi, a)$, it turns out that it closely approximates $E_{R}$, as can be seen in Table $\underline{1}$. Table 1 represents typical energy-optimal flight paths, generated with con-

Table 1 Comparison of $P_{R}$ and $E_{R}$ on an energy-optimal paths

\begin{tabular}{lcc}
\hline \hline$P_{R}$ & $E_{R}$ & Error $\left(P_{R}-E_{R}\right) / E_{R}, \%$ \\
\hline 2.3492 & 2.3511 & -0.08 \\
2.2016 & 2.2125 & -0.49 \\
2.0771 & 2.0745 & 0.13 \\
1.9112 & 1.9307 & -1.01 \\
1.7704 & 1.7822 & -0.66 \\
1.6304 & 1.6226 & 0.48 \\
1.4810 & 1.4548 & 1.80 \\
1.3149 & 1.2983 & 1.28 \\
1.1526 & 1.1551 & -0.22 \\
1.0068 & 1.0041 & 0.27 \\
0.9385 & 0.9496 & -1.17 \\
0.7861 & 0.8180 & -3.9 \\
0.6315 & 0.6864 & -8.00 \\
0.4751 & 0.5470 & -13.14 \\
\hline \hline
\end{tabular}


Table 2 Simulation conditions

\begin{tabular}{lc}
\hline \hline Condition & Value \\
\hline Initial position $\left(x_{o}, y_{o}\right), \mathrm{m}$ & $(0,0)$ \\
Initial energy $E_{o}, \mathrm{~J}$ & 0 \\
Initial heading $\psi_{o}, \mathrm{deg}$ & 127 \\
Final position $\left(x_{f}, y_{f}\right), \mathrm{m}$ & $(700,1300)$ \\
Mission time $T_{M}, \mathrm{~s}$ & 300 \\
Velocity of minimum power $V_{\text {power }_{\text {min }}}, \mathrm{m} / \mathrm{s}$ & 15 \\
Vehicle parameters & {$[53]$} \\
\hline \hline
\end{tabular}

ditions similar to those described in Table 2. Thus, we have the following:

Experimental fact 1: $P_{R}$ can be used instead of $E_{R}$ to determine the regime of the optimal flight.

\section{Properties of Extremal Flight Paths}

From the necessary conditions, we formulate the following propositions.

Proposition 1: If $P_{\mathrm{sd}}$ is sufficiently small, then $\phi(t)=0$ and $V=V_{\text {energy }}$ generate a path that satisfies the necessary conditions for optimal flight, and $t_{f} \leq t_{o}+T_{M}$.

Note that, in practice, small $P_{\text {sd }}$ implies $P_{R}<1$. However, Proposition 1 does not mean that $P_{R}<1$ implies $\phi(t)=0$ and $V=V_{\text {energy }_{\min }}$ generate the only path that satisfies the necessary conditions of optimality. It is possible to find additional paths satisfying the necessary conditions in which $P_{R}<1$ and $\phi(t) \neq 0$ [53].

Proposition 2: If $P_{R}>1$, and $T_{M}$ and $P_{R}$ are sufficiently large, then the optimal path must satisfy $t_{f}=t_{o}+T_{M}$ and $V=V_{\text {power }_{\text {min }}}$.
Proposition 2 implies that when $P_{R}>1$ and is large enough, and $T_{M}$ is large enough, the optimal path takes as much time as allowed, flying at the most advantageous speed. Propositions 1 and 2 are proven in [53], based on Experimental Fact 1.

\section{A. Characteristics of Optimal Paths}

The same set of simulation conditions was used for each result presented in this section. These conditions are shown in Table 2.

Figures $\underline{1}$ and $\underline{2}$ are representative samples of optimal flight paths. Each flight path was then evaluated based upon flight duration and total energy at the end of flight. A summary of the conditions and results for Fig. 1 is presented in Table 3 .

Of particular note in Fig. 1 is the positive value of $E_{\text {total }}$ at the end of flight, indicating a net gain of energy. The flight duration is equal to $T_{M}$. The final time $t_{f}$ is free in this problem subject to $t_{f} \leq t_{o}+T_{M}$. The aircraft also only made a slow, sweeping turn with $|\phi(t)| \ll 1$ at all times. Throughout the flight, the speed remained a constant $15 \mathrm{~m} / \mathrm{s}$. The results from this flight are in accordance with Proposition 2.

A summary of the conditions and results for Fig. 2 is presented in Table 4. The major difference with the previous case is that the sun has set. During this flight, the bank angle is close to 0 deg, indicating almost no turning. The only turn was at the beginning of flight to obtain a direct heading toward the destination. The control inputs during this turn indicate a high bank angle for a short duration. The flight duration was only $80 \mathrm{~s}$, much less than $T_{M}$. In this case, the total energy at the end of the flight was negative, indicating that more energy was lost than collected. The speed throughout the flight remained at a constant $19 \mathrm{~m} / \mathrm{s}$. The results from this flight are in accordance with Proposition 1.

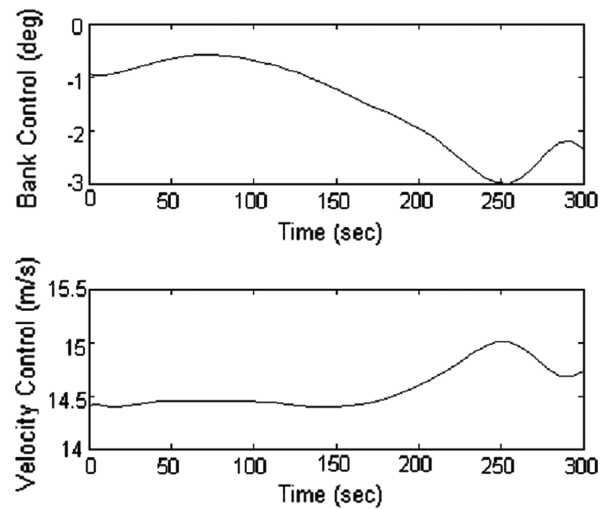

a) Bank angle and velocity
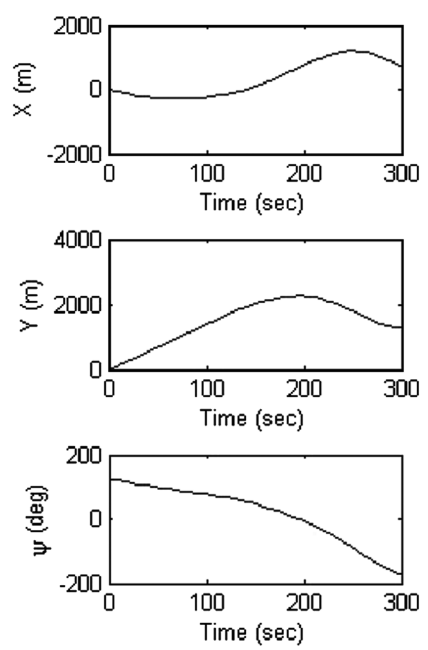

c) States and Costates

Fig. 1 Example of an energy-optimal flight path based on Table 3. Dots are placed every $20 \mathrm{~s}$.

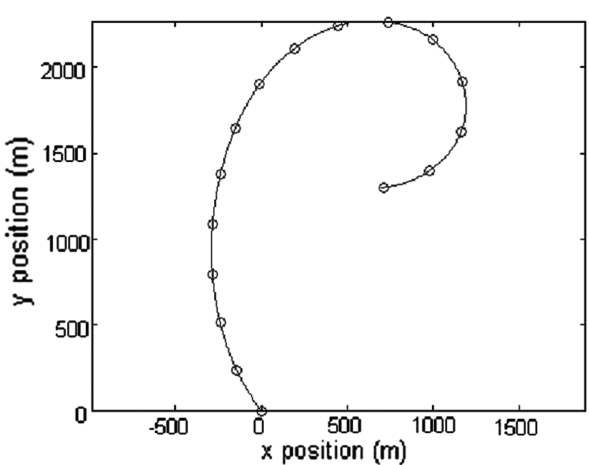

b) Flight path
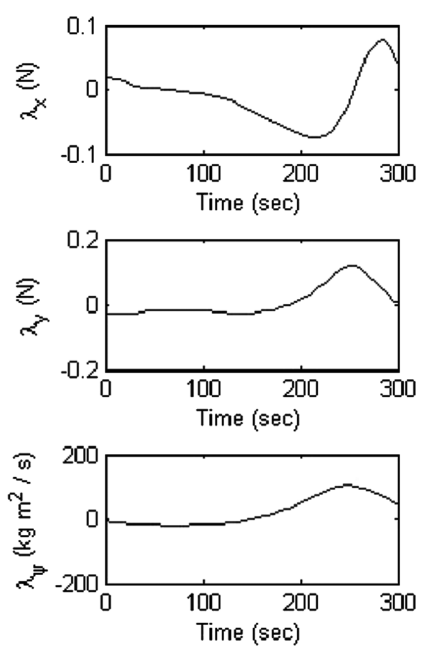


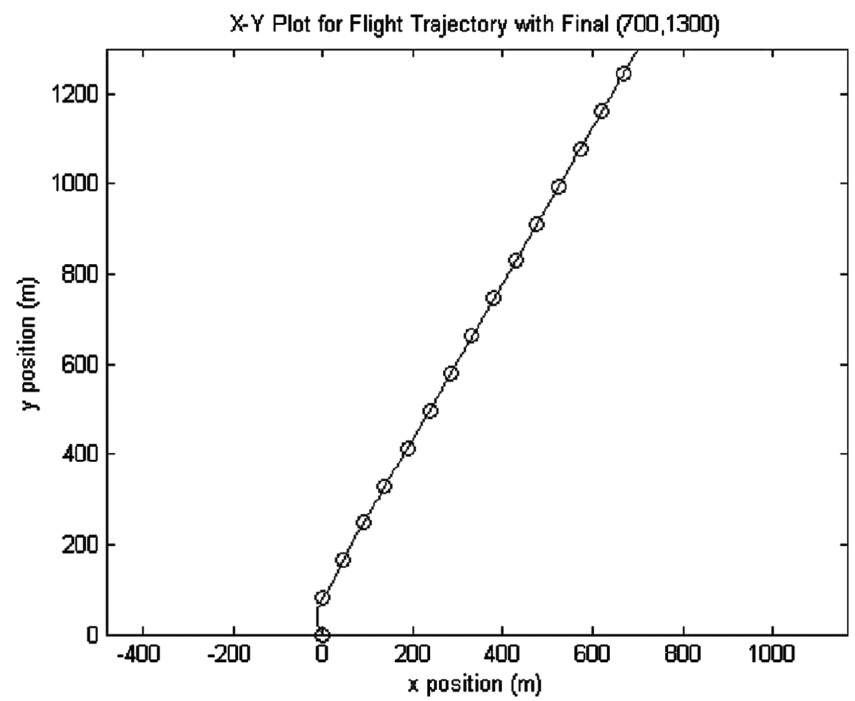

Fig. 2 Example of an energy-optimal flight path based on Table 4 . Dots are placed every $5 \mathrm{~s}$.

As shown in Figs. $\underline{1}$ and $\underline{2}$, the flight-path characteristics vary widely as the elevation is changed. Figure 3 is obtained by varying the elevation of the sun and recording the total energy of the resulting optimal flight path, whereas the remaining aircraft, environmental, and mission parameters are fixed. The elevation is varied from 0 to $90 \mathrm{deg}$. Although the total energy of the aircraft at the end of flight remains positive, a nearly sinusoidal relationship between elevation and energy emerges. This relationship persists until the total energy becomes negative. At this transition point, the trend departs from a nearly sinusoidal function, which suggests a change in regime. The dashed line in the figure represents the total energy acquired during a straight flight directly toward the final position. The transition exhibited in Fig. 3 supports the use of the power ratio as a predictor of the optimal regime.

Disturbances such as wind can affect the flight path of the UAV. These disturbances are considered in [53].

\section{B. Extremal Path Summary}

In summary, extremal flight paths can be obtained as follows. When $P_{R}<1$, extremal flight paths are best described by Proposition 1. When $P_{R}>1$, extremal flight paths satisfy Proposition 2. From Propositions 1 and 2, the velocity in both regimes is not an independent control, but is instead dependent upon the regime and the bank angle.

Table 3 Figure $\underline{1}$ simulation conditions and results

\begin{tabular}{lc}
\hline \hline Condition & Value \\
\hline Sun position $(a, e)$, deg & $(0,45)$ \\
Solar spectral density $P_{\text {sd }}, \mathrm{W} / \mathrm{m}^{2}$ & 380 \\
Final heading $\psi_{f}$, deg & 180 \\
Flight duration $t_{f}-t_{o}, \mathrm{~s}$ & 300 \\
Total final energy $E_{T}, \mathrm{~J}$ & 6764 \\
Energy in $E_{\mathrm{in}}, \mathrm{J}$ & 12,646 \\
\hline \hline
\end{tabular}

Table 4 Figure 2 simulation conditions and results

\begin{tabular}{lc}
\hline \hline Condition & Value \\
\hline Sun position $(a, e)$, deg & $(0,0)$ \\
Solar spectral density $P_{\text {sd }}, \mathrm{W} / \mathrm{m}^{2}$ & 380 \\
Final heading $\phi_{f}$, deg & 61 \\
Flight duration $t_{f}-t_{o}, \mathrm{~s}$ & 80 \\
Total final energy $E_{T}, \mathrm{~J}$ & $-1776.1 \mathrm{~J}$ \\
Energy in $E_{\text {in }}, \mathrm{J}$ & 0 \\
\hline \hline
\end{tabular}

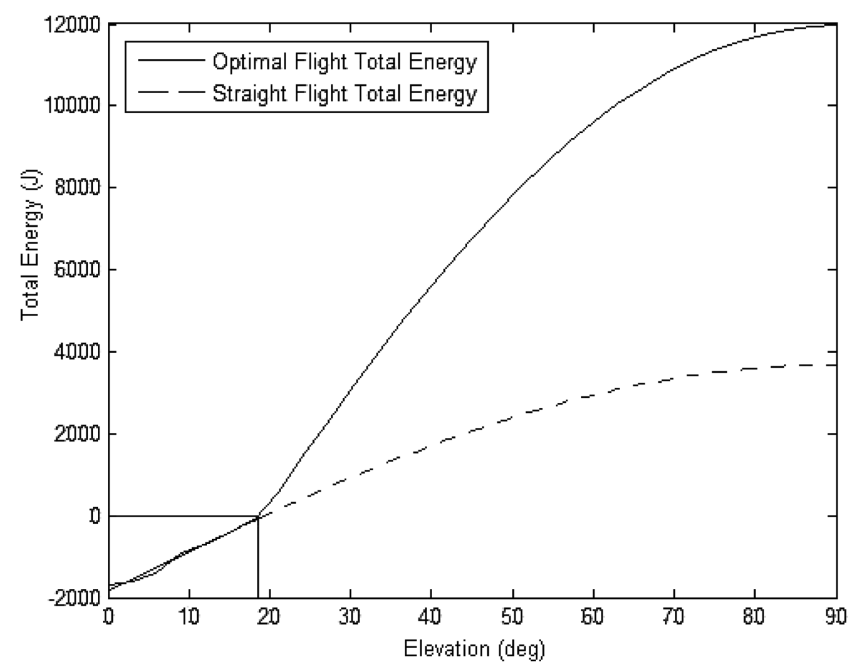

Fig. 3 Total energy at end of flight as a function of solar elevation.

\section{Perpetuity Threshold}

\section{A. Derivation of the Perpetuity Threshold}

For perpetual endurance, it is required that, over the duration of a solar day, the energy collected by the aircraft exceed or be equal to the energy lost: that is,

$$
\frac{E_{\text {in }}}{E_{\text {out }}} \geq 1
$$

From Eqs. (7) and (13), this is equivalent to

$$
\frac{\int_{t_{r_{1}}}^{t_{s}} P_{\text {in }}(\theta) \mathrm{d} t}{\int_{t_{r_{1}}}^{t_{r_{2}}} P_{\text {out }}(\phi, V) \mathrm{d} t} \geq 1
$$

where we need only consider the daylight hours for the power collected. Here, $t_{r_{1}}$ is the time of sunrise on a solar day, $t_{s}$ is the next time of sunset, and $t_{r_{2}}$ is the time of sunrise on the next solar day.

Because the final conditions for loiter are free and banking requires energy (see Remark 1), energy-optimal loitering paths have zero bank angle and a speed equal to $V_{\text {power }_{\text {min }}}$. We use that fact in Eq. (5) and the time invariance of $P_{\text {out }}$ to show that Eq. (41) is equivalent to

$$
\frac{\eta_{\mathrm{sol}} P_{\mathrm{sd}} S \int_{t_{R}}^{t_{S}} \sin (e(t)) \mathrm{d} t}{P_{\text {out }} t_{\mathrm{sd}}} \geq 1
$$

Let $\bar{e}$ be the average elevation of the sun; that is, let $\bar{e}$ satisfy

$$
\sin (\bar{e})=\frac{1}{t_{s}-t_{r}} \int_{t_{r}}^{t_{s}} \sin (e(t)) \mathrm{d} t
$$

We can simplify Eq. (2ㅡ) as

$$
\frac{\eta_{\mathrm{sol}} P_{\mathrm{sd}} S \sin (\bar{e}(t))\left(t_{s}-t_{r}\right)}{P_{\text {out }} t_{\mathrm{sd}}} \geq 1
$$

Comparing this with Eq. (38), this inequality is equivalent to

$$
P_{R}(\bar{e}) \geq \frac{t_{\mathrm{sd}}}{t_{s}-t_{r}}
$$

Hence, Eq. (45) solves the perpetuity problem by establishing the following:

Proposition 3: Perpetual endurance is possible if and only if the power ratio, evaluated at the average sun elevation, exceeds the reciprocal of the daylight duty cycle.

Remark 2: Note that the right-hand side of Eq. (45) is always greater than or equal to 1 . Therefore, perpetual endurance always requires that the power ratio, evaluated at the average sun elevation, be greater than or equal to 1 . 


\section{B. Comparative Analysis of the Perpetuity Thresholds on Earth and Mars}

The results of Sec. VII.A provide a threshold, dependent upon location and time of year, that must be exceeded by the power ratio for loitering flight. Note that design parameters and environmental parameters affect the power ratio. If we fix the design of the aircraft, we can examine the effect of environmental parameters on the power ratio. Furthermore, we can compare the design requirements for perpetual endurance between Earth and Mars.

The perpetuity threshold has been shown to be the ratio $t_{\mathrm{sd}} /$ $\left(t_{s}-t_{r}\right)$. We can compare this ratio, as a function of mean anomaly $\Omega t$ and latitude, between Earth and Mars, as shown in Figs. 4 and $\underline{5}$. Note that the perpetuity threshold approaches infinity when the daylight duty cycle approaches zero, and we have limited the plot to thresholds smaller than 6 . The arctic regions are those latitudes above $90^{\circ}-i$ and below $-90^{\circ}+i$. During part of the year, these regions can have extended periods of darkness (i.e., arctic winter) or light (i.e., arctic summer); that is, there are no local sunrises or sunsets for multiple rotations of the planet. For arctic summer, the power ratio need only exceed 1 for perpetual endurance. We have not considered arctic winter in this paper because requirements on battery size put this case outside the scope of practical solar-powered aircraft.

Table $\underline{5}$ compares the planetary characteristics of Earth and Mars.

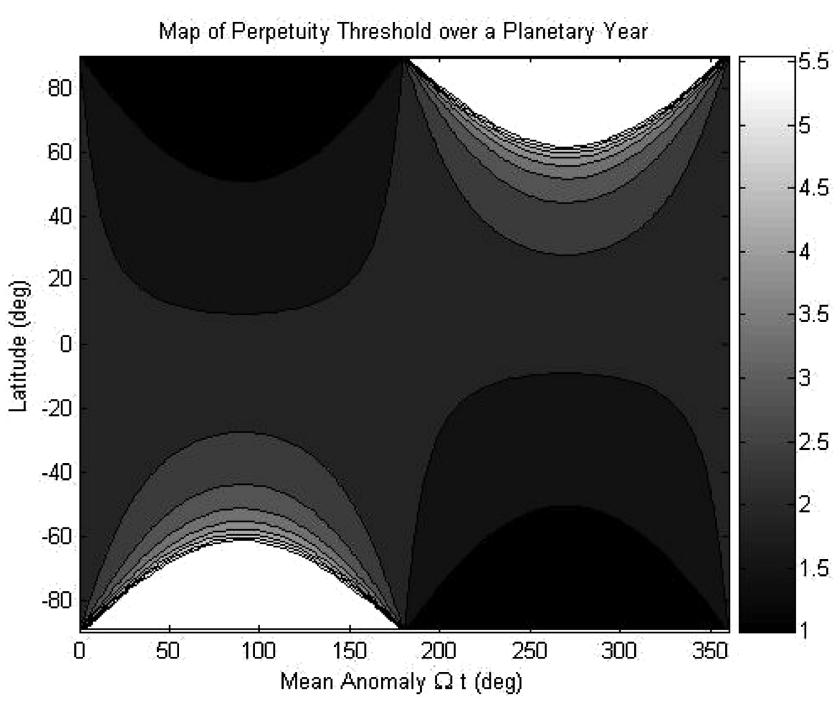

Fig. 4 Map of perpetuity threshold on Earth.

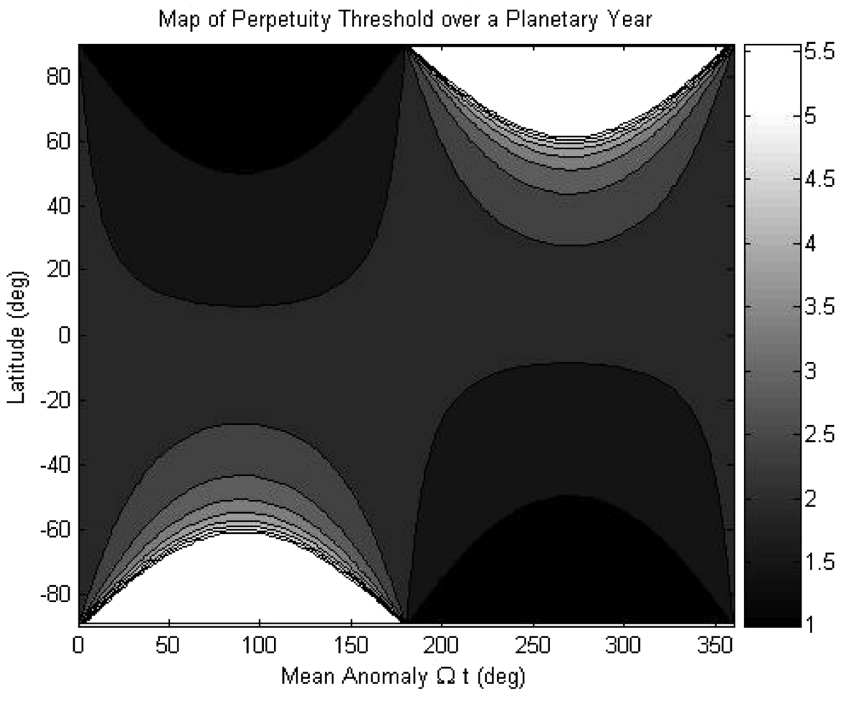

Fig. 5 Map of perpetuity threshold on Mars.
Table 5 Planetary characteristics of Earth and Mars

\begin{tabular}{lcc}
\hline \hline Characteristics & Earth & Mars \\
\hline Duration of solar day $t_{\mathrm{sd}}, \mathrm{h}$ & 23.93 & 24.62 \\
Days in year & 365.25 & 687 \\
Inclination $i$ of axis, deg & 23.5 & 25 \\
\hline \hline
\end{tabular}

The maximum deviation between the thresholds on Earth and Mars is $6.3 \%$, with a mean deviation of $-5 \times 10^{-2} \%$. The perpetuity thresholds between Earth and Mars are therefore similar because the maximum and average deviations are small.

\section{Comparative Analysis of the Power Ratios on Earth and Mars}

The power ratio (38) can be rewritten as

$$
P_{R}=\left[0.402 P_{\mathrm{sd}} \eta_{\mathrm{sol}} \sin (e) \sqrt{\rho} g^{\frac{2}{3}}\right]\left[\sqrt[4]{\frac{\eta_{\mathrm{prop}}^{4} b^{6} \epsilon^{3} \pi^{3} S^{3}}{C_{D_{o}} m^{6}}}\right]
$$

highlighting the separate roles of environmental and aircraft parameters, respectively. Furthermore, if a constant loading and thickness are assumed across the wing planform, we can set $m=\rho_{w} S$, where $\rho_{w}$ is the mass per unit area of the wing. This simplifies Eq. (46) as

$$
P_{R}=\left[0.402 P_{\mathrm{sd}} \eta_{\mathrm{sol}} \sin (e) \sqrt{\rho} g^{\frac{2}{3}}\right]\left[\sqrt[4]{\frac{\eta_{\mathrm{prop}}^{4} \epsilon^{3} \pi^{3} \mathrm{AR}^{3}}{C_{D_{o}} \rho_{w}^{6}}}\right]
$$

which indicates that to increase the power ratio, a low wing density and high aspect ratio should be used. Remarkably, to date, all successful solar-powered aircraft have low wing density and high aspect ratio [4]. The second term in the right-hand side of Eq. (47) elucidates why this is so.

To compare the power ratios of a given aircraft on Earth and Mars, rewrite Eq. (47) as

$$
P_{R}=0.402 \eta_{\mathrm{sol}} \sin (e) \sqrt[4]{\frac{\eta_{\mathrm{prop}}^{4} \epsilon^{3} \pi^{3} \mathrm{AR}^{3}}{\rho_{w}^{6}}}\left(\frac{P_{\mathrm{sd}} \sqrt{\rho}}{g^{\frac{3}{2}} \sqrt[4]{C_{D_{o}}}}\right)
$$

where $P_{\mathrm{sd}}, \rho, g$, and $C_{D_{o}}$ are all determined by the planet. Note that $C_{D_{o}}$ depends on the Reynolds number of the aircraft, which itself depends upon viscosity and atmospheric density. The contribution of $P_{\text {sd }}, \rho, g$, and $C_{D_{o}}$ to the power ratio (48) is the term

$$
\frac{P_{\mathrm{sd}} \sqrt{\rho}}{g^{\frac{3}{2}} \sqrt[4]{C_{D_{o}}}}
$$

for which the values on Earth and Mars can be compared. Table $\underline{6}$ compares the constant environmental parameters $P_{\mathrm{sd}}, \rho$, and $g$ on Earth and Mars.

Note that the quantity $P_{\mathrm{sd}} \sqrt{\rho} / g^{\frac{3}{2}}$ is 4.9 times larger on Earth than on Mars. Reference [55] also compares solar-powered flight on Earth and Mars and reaches a similar conclusion, but without accounting for parasitic drag. We must indeed consider $C_{D_{o}}$ in Eq. (49). Because $C_{D_{o}}$ depends upon velocity, atmospheric density, and viscosity, a comparison of its values on Earth and Mars is not straightforward. Figure 6 illustrates this comparison over a range of speeds. Note that $C_{D_{o}}$ is a lways smaller for Earth than for Mars.

Combining the results of Table 6 and Fig. 6 leads to the following conclusion: The power ratio of an äircraft on Earth is always at least 4.9 times larger than the power ratio of the same aircraft on Mars.

\section{Comparison of Requirements for Perpetual Endurance on Earth} and Mars

The results of Secs. VII.A-VII.C. allow us to make the following general statement: For a given latitude and time of year, it is always easier to design an aircraft to fly perpetually on Earth than on Mars. Several items contribute to this statement: 


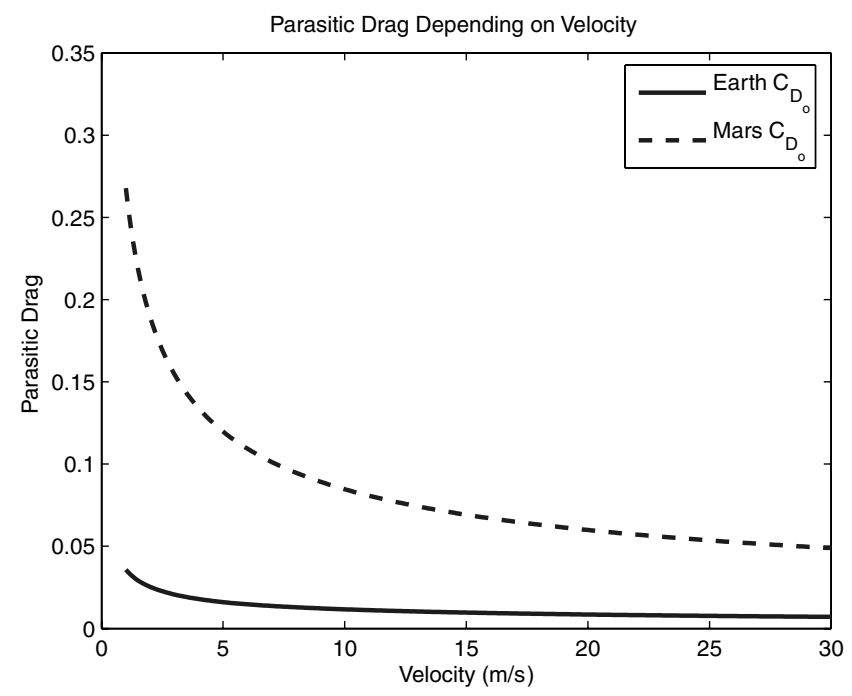

Fig. 6 Comparison of $C_{D_{o}}$ on Earth and Mars.

1) The perpetuity thresholds for a given date and latitude are almost identical on both planets.

2) The contribution of environmental parameters in the power ratio is always at least 4.9 times larger on Earth than on Mars.

\section{E. Examples}

The University of Michigan SolarBubbles Student Team has been designing, building, and testing an aircraft for solar-powered flight. It is named Huitzilopochtli, or Hui for short, after the sun god of the Aztecs, and is a glider-based aircraft used for engineering education and as an autonomous vehicle test platform. Its primary area of flight is near Ann Arbor, Michigan, at a latitude of $42.22^{\circ} \mathrm{N}$ and longitude of $-83.75^{\circ} \mathrm{W}$. We will assume an arbitrary flight date of 6 August.

Reference [53] provides the design parameters for the Hui aircraft. From the analysis in Sec. VII, the power ratio of Hui on Earth is 8.86. Because this power ratio exceeds the perpetuity threshold in Table $\underline{7}$, Hui is capable of perpetual endurance on Earth. Moreover, the power ratio of Hui on Mars is 1.8. Because the perpetuity thresholds on Earth and Mars are very similar, we conclude that the Hui would also be capable of perpetual endurance on Mars.

The Gossamer Penguin was the first manned solar-powered aircraft. Built in 1979 and based upon the Pathfinder solar panel, this aircraft was flown several times across the Mojave desert. The design parameters and assumptions about this aircraft are shown in [53]. If we again compare the power ratio of this aircraft between Earth and Mars, we find that it is 1.03 on Earth and only 0.21 on Mars. Hence,

Table 6 Environmental parameters on Earth and Mars

\begin{tabular}{lcc}
\hline \hline Parameters & Earth & Mars \\
\hline$P_{\mathrm{sd}}, \mathrm{W} / \mathrm{m}^{2}$ & 1353 & 589 \\
$g, \mathrm{~m} / \mathrm{s}^{2}$ & 9.86 & 3.71 \\
$\rho, \mathrm{kg} / \mathrm{m}^{3}$ & 1.29 & 0.015 \\
$P_{\mathrm{sd}} \sqrt{\rho} / \mathrm{g}^{\frac{3}{2}},\left(\mathrm{~kg} / \mathrm{m}^{3}\right)^{\frac{3}{2}}$ & 49.63 & 10.10 \\
\hline \hline
\end{tabular}

Table 7 Perpetuity parameters

\begin{tabular}{lc}
\hline \hline Parameters & Values \\
\hline Mean anomaly $\Omega t$, deg & 136 \\
Duration of solar day $t_{\text {sd }}, \mathrm{h}$ & 24 \\
Duration of daylight $t_{s}-t_{r}, \mathrm{~h}$ & 14.2 \\
Perpetuity threshold $P_{T}$ & 1.7 \\
Average elevation $\bar{e}, \mathrm{deg}$ & 45.95 \\
\hline \hline
\end{tabular}

the Gossamer is capable of solar-powered flight on Earth but would not have that capability on Mars.

The preceding examples illustrate that some solar-powered aircraft are quite capable of perpetual endurance on both Earth and Mars, but others can only fly perpetually on Earth.

\section{F. Effect of Altitude on the Power Ratio}

The threshold of power ratio for perpetual flight depends on date and location. In [42], the maximum altitude that a solar-powered aircraft could achieve without onboard energy storage was found. Here, the effect of altitude on the power ratio, and thus on the endurance of a solar-powered aircraft, is discussed.

As shown in Eq. (48), several parameters of the power ratio are environmental. All of these parameters are dependent upon altitude in some way. Although planets differ, a general statement can be made that $\rho, g$, and $C_{D_{o}}$ decrease with altitude, whereas $P_{\text {sd }}$ increases with altitude.

By taking all the altitude-dependent environmental parameters into account, the total power collected is shown in Fig. 7.

We have shown in [53] that there is a maximum altitude, a so-called solar ceiling, at which solar-powered aircraft can fly and that there is a optimal altitude for the aircraft to fly. These locations cannot be computed analytically but can be found numerically.

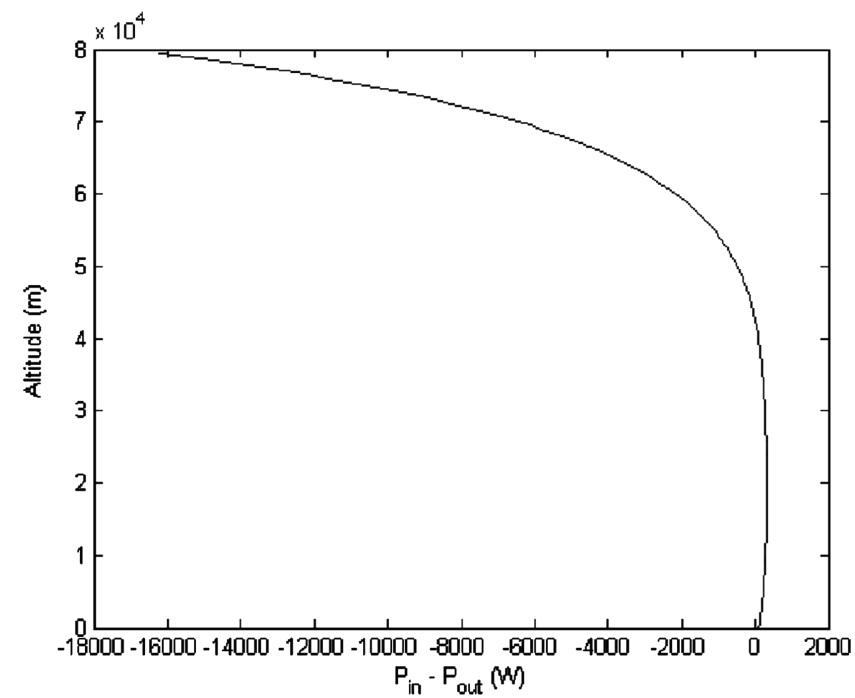

a) Total power

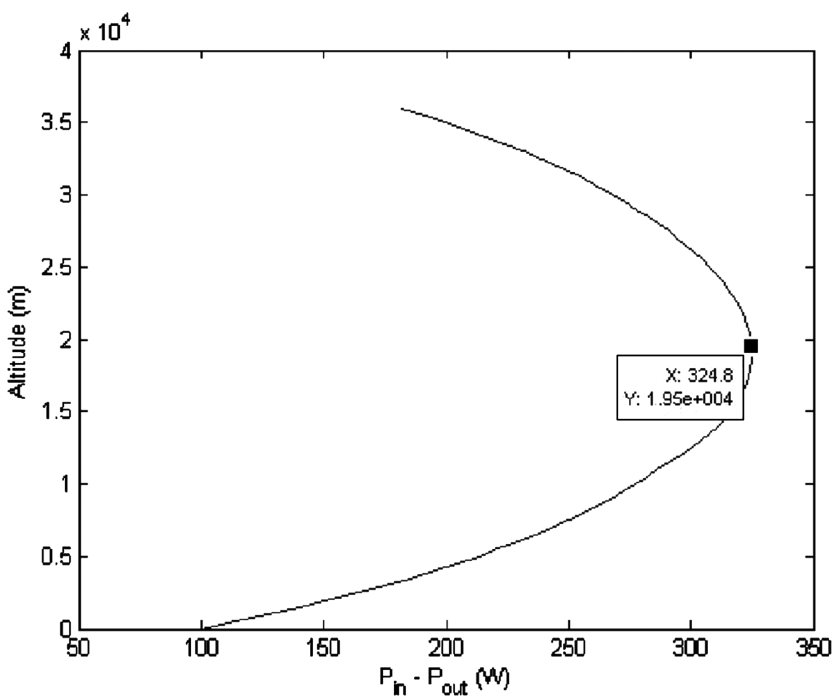

b) Total power (Close-up)

Fig. 7 Total power for Hui at altitude; the best use of power occurs at $15 \mathrm{~km}$ with a gain of $126.6 \mathrm{~W}$. 


\section{Conclusions}

Path planning for solar-powered unmanned aerial vehicles (UAVs) can be improved if a model that couples the kinematics with the energetics through the bank angle of the aircraft is used. By identifying and predicting the regime of optimal flight through use of the power ratio, significant energy savings can be made. In addition, the similarity in looks of solar-powered UAVs is not accidental: analysis of the power ratio shows that it is required for efficient design.

During perpetual flight, a positive total energy balance must be achieved over a solar day. By translating this requirement on the power ratio, we obtain that perpetual solar-powered flight is achievable if and only if the power ratio, evaluated at the average sun elevation, is greater than or equal to the reciprocal of the daylight duty cycle. A comparative analysis of the environmental factors in the power ratio leads to the conclusion that perpetual solar-powered flight is significantly more difficult on Mars than on Earth.

\section{References}

[1] “The Vision for Space Exploration,” NASA, Feb. 2004, http://www. nasa.gov/pdf/55583main_vision_space_exploration2.pdf [retrieved 15 Dec. 2008]

[2] Irving, F. G., and Morgan, D., "The Feasibility of an Aircraft Propelled by Solar Energy," 2nd International Symposium on the Technology and Science of Low Speed and Motorless Flight, AIAA Paper 1974-1042, Sept. 1974.

[3] Hall, D. W., Fortenbach, C. D., Dimiceli, E. V., and Parks, R. W., "A Preliminary Study of Solar Powered Aircraft and Associated Power Trains," NASA CR 3699, Dec. 1983.

[4] Boucher, R. J., "History of Solar Flight," 20th SAE/ASME Joint Propulsion Conference, AIAA Paper 84-1429, June 1984.

[5] Colella, N. J., and Wenneker, G. S., "Pathfinder and the Development of Solar Rechargeable Aircraft," Energy and Technology Review, July 1994, pp. 1-9, https://www.llnl.gov/etr/pdfs/07_94.1.pdf [retrieved 24 Mar. 2009].

[6] Baldock, N., and Mokhtarzadeh-Dehghan, M. R., "A Study of SolarPowered, High-Altitude Unmanned Aerial Vehicles," Aircraft Engineering and Aerospace Technology, Vol. 78, No. 3, 2006, pp. $187-193$.

[7] William H. Phillips, "Some Design Considerations for Solar-Powered Aircraft," NASA TR 1675, June 1980.

[8] MacCready, P. B., Lissaman, P. B. S., Morgan, W. R., and Burke, J. D., "Sun Powered Aircraft Design," Annual Meeting and Technical Display on Frontiers of Achievement, AIAA Paper 1981-916, May 1981.

[9] Youngblood, J. W., and Talay, T. A., "Solar-Powered Airplane Design for Long-Endurance, High Altitude Flight," 2nd International Very Large Vehicles Conference, AIAA Paper 1982-811, May 1982.

[10] Youngblood, J. W., Talay, T. A., and Pegg, R. J., "Design of LongEndurance Unmanned Airplanes Incorporating Solar and Fuel Cell Propulsion," 20th SAE/ASME Joint Propulsion Conference, AIAA Paper 1984-1430, June 1984.

[11] Stella, P. M., and Flood, D. J., "Photovoltaic Options for Solar Electric Propulsion," NASA TM 103284, July 1990.

[12] Brandt, S. A., and Gilliam, F. T., "Design Analysis Methodology for Solar-Powered Aircraft," Journal of Aircraft, Vol. 32, No. 4, 1995, pp. 703-709.

doi: $10.2514 / 3.46780$

[13] Reinhardt, K. C., Lamp, T. R., Geis, J. W., and Colozza, A. J., "SolarPowered Unmanned Aerial Vehicles," 31st Inter-Society Energy Conversion Engineering Conference, Vol. 1, Inst. of Electrical and Electronics Engineers, Piscataway, NJ, Aug. 1996, pp. 41-46.

[14] Trifu, O., and Savu, G., "Unmanned Solar Powered Aerial Surveyor Configured with an Aerodynamic Optimization Procedure," 15th AIAA Applied Aerodynamics Conference, AIAA Paper 1997-2322, June 1997.

[15] Colozza, A. J., Scheiman, D. A., and Brinker, D. J., "GaAs/Ge Solar Powered Aircraft," NASA TM 1998-208652, Oct. 1998.

[16] Flittie, K., and Curtin, B., "Pathfinder Solar-Powered Aircraft Flight Performance," Atmospheric Flight Mechanics Conference and Exhibit, AIAA Paper 1998-4446, Aug. 1998.

[17] Berry, P., "The Sunriser-A Design Study in Solar Powered Flight," 2000 World Aviation Conference, AIAA Paper 2000-5507, Oct. 2000.

[18] Wilson, C., Nutbean, J., and Bond, I., "Aerodynamic and Structural Design of a Solar-Powered Micro Unmanned Air Vehicle," Proceedings of the Institution of Mechanical Engineers, Part G
(Journal of Aerospace Engineering), Vol. 214, No. 2, 2000, pp. 97-106. doi:10.1243/0954410001531845

[19] Frulla, G., "Preliminary Reliability Design of a Solar-Powered High-Altitude Very Long-Endurance Unmanned Air Vehicle," Proceedings of the Institution of Mechanical Engineers, Part $G$ (Journal of Aerospace Engineering), Vol. 216, No. 4, 2002, pp. 189196.

doi: $10.1243 / 09544100260369722$

[20] Patel, C. K., Arya, H., and Sudhakar, K., "Design, Build and Fly a Solar Powered Aircraft," International Seminar and Annual General Meeting of the Aeronautical Society of India, Aeronautical Society of India, Hyderabad, India, 2002.

[21] Roberts, C., Vaughan, M., and Bowman, W. J., "Development of a Solar Powered Micro Air Vehicle," 40th Aerospace Sciences Meeting and Exhibit, AIAA Paper 2002-703, Jan. 2002.

[22] Dornheim, M. A., "Get Me Through the Night," Aviation Week and Space Technology, Vol. 159, No. 11, Sept. 2003, pp. 66, 68, 70.

[23] Colozza, A., "Solid State Aircraft," NASA Inst. for Advanced Concepts, Atlanta, Nov. 2002.

[24] Noth, A., Engel, W., and Siegwart, R., "Design of an Ultra-Lightweight Autonomous Solar Airplane for Continuous Flight," Field and Service Robotics, edited by P. Corke and S. Sukkariah, Vol. 25, Springer, Berlin, 2006.

[25] Theodore, C. R., Tischler, M. B., and Colbourne, J. D., "Rapid Frequency-Domain Modeling Methods for Unmanned Aerial Vehicle Flight Control Applications," Journal of Aircraft, Vol. 41, No. 4, Aug. 2004, pp. 735-743. doi: $10.2514 / 1.4671$

[26] Dornheim, M. A., "So Long Solar-Powered Drone Stays Aloft for $48 \mathrm{Hr}, "$ Aviation Week and Space Technology, June 2005.

[27] Romeo, G., Frulla, G., Cestino, E., and Borello, F., "SHAMPO: Solar HALE Aircraft for Multi Payload and Operations," Aerotecnica Missili e Spazio, Vol. 85, Sept. 2005.

[28] "Solar-Power Research and Dryden," http://www.nasa.gov/centers/ dryden/news/FactSheets/FS-054-DFRC.html [retrieved 20 Dec. 2008].

[29] Romeo, G., and Frulla, G., "HELIPLAT: Aerodynamic and Structural Analysis of HAVE Solar Powered Platform," 1st Technical Conference and Workshop on Unmanned Aerospace Vehicles, AIAA Paper 20023504, May 2002.

[30] Bryson, A. E., Jr., and Ho, Y.-C., Applied Optimal Control, Hemisphere, New York, 1975.

[31] Barman, J., and Erzberger, H., "Fixed-Range Optimum Trajectories for Short-Haul Aircraft," Journal of Aircraft, Vol. 13, No. 10, 1976, pp. $748-754$. doi: $10.2514 / 3.58706$

[32] Calise, A., and Moerder, D., "Singular Perturbation Techniques for Real Time Aircraft Trajectory Optimization and Control," NASA Scientific and Technical Information Branch, CR 3597, 1982.

[33] Speyer, J., "Nonoptimality of the Steady-State Cruise for Aircraft," AIAA Journal, Vol. 14, No. 11, 1976, pp. 1604-1610. doi: $10.2514 / 3.7257$

[34] Burrows, J. W., "Fuel Optimal Aircraft Trajectories with Fixed Arrival Times," Guidance and Control Conference, AIAA Paper 1981-1749, Aug. 1981.

[35] Sachs, G., and Christodoulou, T., "Reducing Fuel Consumption of Subsonic Aircraft by Optimal Cyclic Cruise," Journal of Aircraft, Vol. 24, No. 9, 1987, pp. 616-622. doi: $10.2514 / 3.45486$

[36] Schultz, R., and Zagalsky, N., "Aircraft Performance Optimization," Journal of Aircraft, Vol. 9, No. 2, 1972, pp. 108-114. doi: $10.2514 / 3.44326$

[37] Fan, Y., Lutze, F., and Cliff, E., "Time-Optimal Lateral Maneuvers of an Aircraft," Journal of Guidance, Control, and Dynamics, Vol. 18, No. 5, 1995, pp. 1106-1112. doi: $10.2514 / 3.21511$

[38] Vian, J., and Moore, J., "Trajectory Optimization with Risk Minimization for Military Aircraft," Journal of Guidance, Control, and Dynamics, Vol. 12, No. 3, 1989, pp. 311-317. doi: $10.2514 / 3.20410$

[39] Clements, J., "Minimum-Time Turn Trajectories to Fly-to Points," Optimal Control Applications and Methods, Vol. 11, No. 1, 1990, pp. 39-50. doi:10.1002/oca.4660110104

[40] Grimm, W., Well, K., and Oberle, H., "Periodic Control for Minimum Fuel Aircraft Trajectories," Journal of Guidance, Control, and Dynamics, Vol. 9, No. 2, 1986, pp. 169-174. doi: $10.2514 / 3.20086$

[41] Menon, P., Kim, E., and Cheng, V., "Optimal Trajectory Planning for Terrain Following Flight," Journal of Guidance, Control, and 
Dynamics, Vol. 14, No. 4, 1991, pp. 807-813. doi: $10.2514 / 3.20716$

[42] Colozza, A. J., "Effect of Date and Location on Maximum Achievable Altitude for a Solar Powered Aircraft," NASA CR 202326, Mar. 1997.

[43] Hall, D. W., Watson, D. A., Tuttle, R. P., and Hall, S. A., "Mission Analysis of Solar Powered Aircraft," NASA CR 172583, July 1985.

[44] Colozza, A. J., "Effect of Power System Technology and Mission Requirements on High Altitude Long Endurance Aircraft," NASA CR 194455, Feb. 1994.

[45] Brown, W., "The History of Power Transmission by Radio Waves," IEEE Transactions on Microwave Theory and Techniques, Vol. 32, No. 9, Sept. 1984, pp. 1230-1242. doi:10.1109/TMTT.1984.1132833

[46] Chichka, D. F., and Speyer, J. L., "Solar-Powered, FormationEnhanced Aerial Vehicle Systems for Sustained Endurance," 1998 American Control Conference, Inst. of Electrical and Electronics Engineers, New York, June 1998.

[47] Colozza, A. J., "Convective Array Cooling for a Solar Powered Aircraft," NASA CR 212084, Jan. 2003.

[48] Allen, M. J., "Updraft Model for Development of Autonomous Soaring Uninhabited Air Vehicles," 44th AIAA Aerospace Sciences Meeting and Exhibit, AIAA Paper 2006-1510, 1996.
[49] Allen, M. J., "Autonomous Soaring for Improved Endurance of a Small Uninhabited Air Vehicle," Proceedings of the 43rd Aerospace Sciences Meeting, AIAA Paper 2005-1025, 2005.

[50] Allen, M. J., "Guidance and Control of an Autonomous Soaring UAV," NASA TM 214611, Feb. 2007

[51] Qi, Y., and Zhao, Y., "Energy-Efficient Trajectories of Unmanned Aerial Vehicles Flying Through Thermals," Journal of Aerospace Engineering, Vol. 18, No. 2, Apr. 1996, pp. 84-92.

[52] Metzger, D. E., and Hedrick, J. K., "Optimal Flight Paths for Soaring Flight," Proceedings of the 2nd International Symposium on the Technology and Science of Low Speed and Motorless Flight, AIAA, New York, 1974, pp. 867-871.

[53] Klesh, A., and Kabamba, P., "Solar-Powered Aircraft: Energy-Optimal Path Planning and Perpetual Endurance," Univ. of Michigan Control Group, Rept. CGR 09-01, Ann Arbor, MI, Jan. 2009.

[54] Anderson, J. D., Aircraft Performance and Design, McGraw-Hill, New York, June 1999.

[55] Noth, A., Engel, W., and Siegwart, R., "Flying Solo and Solar to Mars - Global Design of a Solar Autonomous Airplane for Sustainable Flight," IEEE Robotics and Automation Magazine, Vol. 13, No. 3, Sept. 2006, pp. 44-52. doi:10.1109/MRA.2006.1678138 